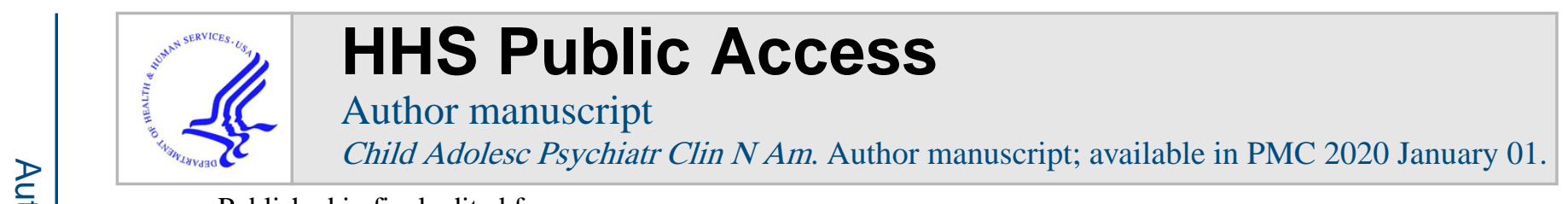

Published in final edited form as:

Child Adolesc Psychiatr Clin N Am. 2019 January ; 28(1): 33-43. doi:10.1016/j.chc.2018.07.003.

\title{
Transcranial Magnetic Stimulation for Adolescent Depression
}

Paul E. Croarkin, DO, MS and

Associate Professor of Psychiatry and Psychology, Division Chair, Child and Adolescent

Psychiatry, Mayo Clinic College of Medicine and Science, Rochester, MN, USA

Frank P. MacMaster, PhD

Associate Professor of Pediatrics and Psychiatry, Scientific Director, Strategic Clinical Network for Addictions and Mental Health, University of Calgary, Alberta Children's Hospital, Calgary, Alberta, Canada

\section{SYNOPSIS}

Adolescent depression is a substantial global public health problem that contributes to academic failure, occupational impairment, deficits in social functioning, substance use disorders, teen pregnancy, and completed suicide. Existing treatment options often have suboptimal results and uncertain safety profiles. Transcranial magnetic stimulation may be a promising, brain-based intervention for adolescents with depression. Existing work has methodological weaknesses and larger, neurodevelopmentally informed studies are urgently needed. Treatment with transcranial magnetic stimulation may modulate cortical GABAergic and glutamatergic imbalances. Future study could inform dosing approaches for TMS based on GABAergic and glutamatergic biomarkers.

\section{Keywords}

adolescent; brain stimulation; depression; GABA; glutamate; neuromodulation; transcranial magnetic stimulation; TMS

\section{Introduction}

Adolescent Major Depressive Disorder (MDD) is a major public health problem with a lifetime prevalence estimated as high as $14-20 \%$ in epidemiological studies. ${ }^{1}$ Worldwide,

\footnotetext{
CORRESPONDING AUTHOR: Paul E Croarkin, DO, MS, 200 First Street SW, Rochester, MN 55905, USA (507) 293-2557, croarkin.paul@mayo.edu.

DISCLOSURE STATEMENT

Dr. Croarkin has received research grant support from Pfizer, Inc., NIMH, the Brain and Behavior Research Foundation, and the Mayo Clinic Foundation. He has served as a site sub principal or principal investigator (without additional compensation) for Eli Lilly and Co., Forest Laboratories, Inc., Merck \& Co., Inc., and Pfizer, Inc.; has received equipment support from Neuronetics, Inc.; and receives supplies and genotyping services from Assurex Health, Inc. for an investigator-initiated study. He is the primary investigator for a multicenter study funded by Neuronetics, Inc. He is a site investigator for a study funded by NeoSync, Inc. Dr. MacMaster has received research grant support from the Canadian Institutes for Health Research, the Canadian Foundation for Innovation, Branch Out Neurological Foundation, Alberta Health Services, and the Alberta Children's Hospital Foundation.

Publisher's Disclaimer: This is a PDF file of an unedited manuscript that has been accepted for publication. As a service to our customers we are providing this early version of the manuscript. The manuscript will undergo copyediting, typesetting, and review of the resulting proof before it is published in its final citable form. Please note that during the production process errors may be discovered which could affect the content, and all legal disclaimers that apply to the journal pertain.
} 
MDD is a leading cause of disease burden. ${ }^{2,3}$ Adolescent depression frequently involves a profound biologic component and ensuing delayed recovery, frequent recurrences, comorbidity, substance abuse, and increased risk for suicide. ${ }^{1,4}$ Initial management of moderate to severe MDD in adolescents involves treatment with psychotherapy and selective serotonin reuptake inhibitors (SSRIs). ${ }^{5,6}$ Remission rates and outcomes are often poor as this treatment does not target relevant, underlying adolescent pathophysiology. ${ }^{5,7}$ Ongoing controversy regarding the effectiveness and safety of SSRIs in young individuals underscores the importance for an improved understanding of the biological mechanisms involved in adolescent depression. ${ }^{8,9}$ Finally, access to evidence based psychotherapy is often limited. ${ }^{10}$

Transcranial magnetic stimulation (TMS) has increasingly been considered as an investigational treatment for adolescents with depression who do not respond to standard treatment modalities such as cognitive behavioral therapy and SSRIs. ${ }^{11,12}$ Treatment with TMS involves the stimulation of cortical neurons with magnetic pulses and is now widely available as a clinical treatment for adults. Current FDA cleared TMS treatments involve 5 daily treatments per week, for 4-6 weeks, with $10 \mathrm{~Hz}, 120 \%$ motor threshold stimulation applied to the left dorsolateral prefrontal cortex. ${ }^{13-15}$ Early adolescent research was informed by this approach but there is a formidable parameter space (for example, coil location, frequency, intensity, duration of treatment, concurrent interventions, and brain state during treatment) to consider for TMS treatment. ${ }^{16,17}$ The heterogeneity of depression in adolescents arguably presents an added layer of complexity. ${ }^{1,16,18}$

\section{Types of TMS}

Early therapeutic TMS research and clinical practice for depression in adults has largely utilized low frequency $(1 \mathrm{~Hz})$ or high frequency $(5-20 \mathrm{~Hz})$ stimulation over the dorsolateral prefrontal cortex..$^{13,19}$ There have been a variety of variations in dosing with time to include deep TMS, accelerated protocols, synchronized TMS, priming protocols, and patterned stimulation. ${ }^{20}$ More contemporary work has examined theta burst stimulation (TBS) dosing strategies as potentially more efficient and durable pulse sequences for the modification of cortical activity. ${ }^{21}$ Treatment with TBS holds the promise of reducing the time burden of treatment for patients. TBS sequences deliver groups of three high frequency pulses $(50 \mathrm{~Hz})$ with interstimulus intervals of $200 \mathrm{~ms}(5 \mathrm{~Hz})$. There are 2 primary TBS patterns that are thought to have discordant neurophysiological effects. ${ }^{21,22}$ Continuous theta burst stimulation (cTBS) involves the delivery of TBS pulses without interruption (typically 2040 seconds $300-600$ pulses) and is thought to decrease cortical excitability. ${ }^{22}$ Intermittent theta burst stimulation (iTBS) delivers 2 second trains of TBS (30 pulses) every 10 seconds and is thought to increase cortical excitability. ${ }^{22,23}$ Recent work in adults with treatment resistant depression suggests that iTBS may be equivalent to standard $10 \mathrm{~Hz}$ rTMS in terms of effectiveness, safety, and tolerability. ${ }^{24}$

\section{Studies of TMS in Depressed Adolescents}

Table 1 summarizes existing therapeutic studies of TMS for adolescents with depression. At present there are 10 publications describing the treatment of 112 unique participants. ${ }^{12,25-33}$ 
Existing literature is almost entirely comprised of case reports and open-label studies. The 2006 study by Loo and colleagues ${ }^{26}$ describes a randomized controlled trial. However, the results from 2 participants assigned to active TMS treatment are all that is described in the publication. ${ }^{26}$ The study by Lee and colleagues ${ }^{30}$ describes the treatment of 25 children with Tourette syndrome. These participants did not have a diagnosis of MDD at baseline.

However, depressive symptoms were tracked and demonstrated group level improvement over the course of TMS treatment. ${ }^{30}$ This study is also unique and important to consider as it examined $1 \mathrm{~Hz}$ TMS which has not been adequately studied in child and adolescent populations with psychiatric disorders. ${ }^{11}$ Otherwise, the majority of studies examined $10 \mathrm{~Hz}$ TMS sessions with protocols adapted from landmark adult studies of TMS. ${ }^{12,14,15,26-28,31,34}$ Farzan and colleagues ${ }^{32}$ have pioneered work with iTBS in adolescents and young adults. Given the increased efficiency of iTBS in terms of both delivery and potential impact on synaptic plasticity, this line of research is critical for future optimization of TMS protocols involving adolescents. ${ }^{32,35}$

\section{Safety}

Systematic data on the safety of TMS in children and adolescent are lacking. ${ }^{11,36}$ While, generally considered safe, TMS interventions could have divergent tolerability and safety profiles across various stages of neurodevelopment. Common concerns include the rare risk of seizure induction, adverse neurocognitive effects, new or exacerbated psychiatric symptoms (such as increased suicidality, hypomania, or mania), aberrant alterations in neuroplasticity, and pain related to the procedure. ${ }^{36}$ Recent, erudite commentaries have highlighted these concerns and the depth of existing knowledge gaps..$^{37,38}$

Krishnan and colleagues ${ }^{36}$ recently reviewed existing literature focused on both TMS and transcranial current stimulation. The review included data from 35 publications focused on the use of TMS in children and adolescents 3-18 years of age. There were very few reported adverse events or tolerability problems among the 322 participants undergoing TMS procedures. Four of these participants (1.2\%) had a major negative side effect. Two participants $(0.62 \%)$ had a seizure and two other participants had syncopal episodes $(0.62 \%)$. Minor side effects such as headache $(11.5 \%)$ and scalp pain $(2.5 \%)$ were described as short-lived and typically resolved without intervention or with the use of over the counter nonsteroidal anti-inflammatory drugs. Other reported adverse events included musculoskeletal problems, twitching, and fatigue. These effects were described as mild and transitory. These data are encouraging and suggest that TMS is relatively safe and tolerable in children and adolescents with appropriate precautions. However, existing work also underscores that in a majority of instances, systematic adverse effect and tolerability data from TMS exposure in children and adolescents are not collected. Systematic, long-term, follow-up studies are also lacking. ${ }^{36}$

The clinical effects and safety of TMS have been examined in numerous other publications. ${ }^{20}$ Published guidelines have been successful in ensuring subject safety. ${ }^{39-41}$ In most cases TMS cannot be applied to individuals with metal in their head (except the mouth). The greatest safety concern is the potential of inducing a seizure. The risk of this is small even with rTMS. The incidence of this has been estimated as no greater than 0.1 to $0.6 \%$ (or 1-6 
in 1,000), which is comparable to the incidence of spontaneous seizures in patients taking antidepressant medications. ${ }^{42}$ In cases in which seizures have been induced in participants, these individuals have recovered with no recurrences. ${ }^{36,39,43}$ There are 3 prior reported seizures in adolescents receiving TMS. ${ }^{44-46}$ In 2 instances the participants were concurrently taking epileptogenic medications (sertraline and olanzapine). ${ }^{44,45}$ One of these participants had also consumed large amounts of alcohol prior to the TMS session (a reported $0.20 \%$ blood alcohol level 30 minutes after the seizure) ${ }^{44}$ In another instance a depressed patient with no risk factors had a seizure with the application of deep TMS. ${ }^{46}$ Presently, it is not clear if the risk for seizure induction during TMS with adolescents is different from that of adults. ${ }^{43}$

\section{Recent Studies}

In 2015, NeuroStar Advanced Therapy ${ }^{\circledR}$ launched the largest, randomized controlled trial of TMS for adolescents (12-21 years of age) with MDD to date. ${ }^{47}$ This trial is scheduled to conclude in late 2018 and will examine the safety and efficacy of NeuroStar TMS $®$ in approximately 100 adolescent participants. The protocol is a randomized, sham-controlled, triple-masked design for the acute treatment of MDD, with a subsequent open-label phase and posttreatment follow-up study. Eligible patients are adolescents aged 12-21 with MDD that has failed to respond to at least 1 but not more than 4 prior antidepressant trials. Phase I offers 6 weeks of either active $10 \mathrm{~Hz}$ TMS or sham treatment applied to the left dorsolateral prefrontal cortex. Phase II provides 6 weeks of open-label $10 \mathrm{~Hz}$ TMS to patients who did not receive protocol-defined clinical benefit in Phase I. Patients with protocol-defined clinical benefit in Phase I or II are eligible for Phase III, a 6-month follow-up study that provides retreatment with TMS for the re-emergence of depressive symptoms. The protocol and study will provide the largest data set to date for the examination of tolerability, safety, and clinical effects of $10 \mathrm{~Hz}$ TMS for MDD in adolescents. ${ }^{47}$

\section{Future Directions}

Neurostimulation technologies such as TMS have great potential as enduring, brain-based interventions for depression in adolescents. ${ }^{11,35}$ Treatment with rTMS likely addresses pathologic imbalances in cortical GABAergic inhibitory and excitatory glutamatergic frontolimbic neurocircuitry. ${ }^{35,48}$ However, at present there are many unknowns regarding optimal stimulation parameters and potential biomarkers for depressed adolescents receiving TMS. ${ }^{11,16}$ Later this year, a National Institute of Mental Health funded, dose-finding, biomarker validation, and effectiveness study of $1 \mathrm{~Hz}$ vs. $10 \mathrm{~Hz}$ TMS for adolescents with depression will begin enrollment with the aim of addressing these questions (NIMH R01MH113700). ${ }^{49}$

Imbalances in GABAergic and glutamatergic tone play a key role in depression, ${ }^{50,51}$ pathophysiologic stress responses, ${ }^{52,53}$ and emotional numbing or anhedonia found in behavioral manifestations of the negative valence system. ${ }^{54}$ These GABAergic and glutamatergic imbalances have differential causes, effects, and behavioral manifestations in adolescents as compared to adults. ${ }^{50,54-57}$ For example, recent preclinical work has demonstrated that repeated stress in adolescent rats inhibits GABAergic projections to the 
amygdala thereby impairing regulatory neurocircuitry. ${ }^{58}$ In adult rats, chronic stress facilitates glutamatergic excitatory neurotransmission with ensuing effects on the lateral nucleus of the amygdala, hippocampus, and frontal cortex. ${ }^{58-62}$ Developmental differences in frontolimibic GABAergic and glutamatergic tone may underlie variances in adolescent depressive symptom presentations and treatment responsivity. ${ }^{54,62}$ A deeper understanding of frontolimbic GABAergic and glutamatergic tone in adolescent depression would assist with precision medicine approaches and intervention development. ${ }^{16}$ Transcranial magnetic stimulation (TMS) and magnetic resonance spectroscopy (MRS) provide complementary measures or cortical GABAergic and glutamategeric tone. ${ }^{63-66}$ Single and paired-pulse TMS paradigms are used to study the physiology of the brain. Neurophysiological measures collected with transcranial magnetic stimulation (TMS) such as intracortical facilitation (ICF), short-interval intracortical inhibition (SICI), long-interval intracortical inhibition (LICI), and the cortical silent period (CSP) are noninvasive measures of cortical GABAergic and glutamatergic tone. ${ }^{65-67}$ Prior work suggests that ICF is a valid marker of glutamatergic tone and it may have utility as a biomarker for depression in adolescents. ${ }^{56,68}$ Ultra-high field, 7 tesla (7 T) MRS adequately quantifies GABA, glutamate, and glutamine concentrations in the cortex for complementary data examining GABAergic and glutamatergic tone. ${ }^{69}$ Concurrent measures with TMS neurophysiological paradigms and $7 \mathrm{~T}$ MRS would provide a refined understanding of GABAergic and glutamatergic tone in disorders of the negative valence systems and mechanistic studies of brain stimulation treatments such as TMS. ${ }^{69,70}$

Figure 1 summarized the protocol of the pending study. Participants in Phase I will be stratified based on ICF testing (high or low) at baseline. An ICF of $>1.5$ at baseline is considered "high" and an ICF $\leq 1.5$ is considered "low". After stratification, adolescents are randomized to either LDLPFC $1 \mathrm{~Hz}$ rTMS with 2400 continuous pulses per session at $120 \%$ motor threshold or LDLPFC $10 \mathrm{~Hz}$ rTMS with 4 seconds on 36 seconds off for 2400 pulses each session at $120 \%$ of resting motor threshold. Hence sessions in each treatment arm with two different types of rTMS $(1 \mathrm{~Hz}$ and $10 \mathrm{~Hz}$ ) will have identical intensities $(120 \%$ motor threshold) durations (40 minutes), number of pulses (2400), and treatment location (LDLFPC). Participant non-responders in Phase I will be offered to the opportunity to enroll in a Phase II. Participants will be undergo therapeutic rTMS sessions with a Neurostar XPLOR system ${ }^{\circledR}$ magnetic stimulator. The research team will localize rTMS treatment sites with the Beam F3 method. ${ }^{71}$ Prior research demonstrates that this is a valid and reliable method for scalp location of the dorsolateral prefrontal cortex with comparable results to more expensive, time intensive, MRI-guided approaches. ${ }^{72}$ Our prior research demonstrates that the Beam F3 method is a feasible and reliable method for rTMS treatment localization in adolescents. ${ }^{12}$ Efficacy measures (Children's Depression Rating Scale Revised [CDRS$\mathrm{R}])^{73}$ and TMS biomarkers will be collected at baseline and weekly. The TMS biomarker panel includes ICF, Motor threshold (MT) Short-interval intracortical inhibition (SICI), long-interval intracortical inhibition (LICI), and cortical silent period (CSP). Adolescent participants receiving TMS will have the opportunity to undergo pre and post $7 \mathrm{~T}$ MRS scans to collect cortical GABA, glutamate, and glutamine levels. ${ }^{49}$

Participants in Phase II will be assigned to 2 weeks of cTBS if their intracortical facilitation measure (baseline assessment for TBS extension trial) is $>1.5$. Participants will be assigned 
to 2 weeks of iTBS if their ICF (baseline assessment for TBS extension trial) is $<1.5$. Extension trial TBS will be applied to the LDLPFC with the Beam F3 method. Participants receiving cTBS will receive 10 daily ( 5 sessions per week for two weeks) 120 second trains of uninterrupted TBS for 1800 pulses at $80 \%$ motor threshold. Participants receiving iTBS will receive 10 daily ( 5 sessions per week for two weeks) 2 second trains every 10 seconds for a total of 570 seconds for 1800 pulses at $80 \%$ motor threshold. Efficacy measures (CDRS-R) and TMS biomarkers will be collected at baseline, 1 week, and 2 weeks. The TMS biomarker panel includes ICF, Motor threshold (MT) Short-interval intracortical inhibition (SICI), long-interval intracortical inhibition (LICI), and cortical silent period (CSP). ${ }^{49}$

\section{Summary}

Safe, effective, brain-based treatments for depression in adolescents could alleviate substantial morbidity and mortality. ${ }^{16}$ Early investigational TMS for adolescent depression is promising. ${ }^{11,36}$ These data suggest that the clinical effects, safety, and tolerability of TMS in adolescents may be similar to what has been described in adults. ${ }^{11,36}$ However, enthusiasm must be tempered by considerations for neurodevelopment and the unknowns associated with TMS exposure in adolescents. ${ }^{16,37,38}$ Larger studies will soon provide more systematic data to examine the clinical tolerability, safety, and clinical effects of TMS in adolescents with depression. ${ }^{47}$ Planned dose-finding and biomarker development studies hold the prospect of expanding the knowledge base of TMS use in depressed adolescents, the pathophysiology of depression in youth, and how TMS modulates cortical GABAergic and glutamatergic neurochemistry. ${ }^{49}$

\section{References}

1. Thapar A, Collishaw S, Pine DS, Thapar AK. Depression in adolescence. Lancet. 2012; 379(9820): 1056-1067. [PubMed: 22305766]

2. Gore FM, Bloem PJ, Patton GC, et al. Global burden of disease in young people aged 10-24 years: a systematic analysis. Lancet. 2011; 377(9783):2093-2102. [PubMed: 21652063]

3. Whiteford HA, Degenhardt L, Rehm J, et al. Global burden of disease attributable to mental and substance use disorders: findings from the Global Burden of Disease Study 2010. Lancet. 2013; 382(9904):1575-1586. [PubMed: 23993280]

4. Naicker K, Galambos NL, Zeng Y, Senthilselvan A, Colman I. Social, demographic, and health outcomes in the 10 years following adolescent depression. The Journal of adolescent health: official publication of the Society for Adolescent Medicine. 2013; 52(5):533-538. [PubMed: 23499382]

5. March JS, Silva S, Petrycki S, et al. The Treatment for Adolescents With Depression Study (TADS): long-term effectiveness and safety outcomes. Archives of general psychiatry. 2007; 64(10):11321143. [PubMed: 17909125]

6. Kennard BD, Emslie GJ, Mayes TL, et al. Sequential treatment with fluoxetine and relapse-prevention CBT to improve outcomes in pediatric depression. Am J Psychiatry. 2014; 171(10): 1083-1090. [PubMed: 24935082]

7. Walkup JT. Antidepressant Efficacy for Depression in Children and Adolescents: Industry- and NIMH-Funded Studies. Am J Psychiatry. 2017; 174(5):430-437. [PubMed: 28253735]

8. Hammad TA, Laughren T, Racoosin J. Suicidality in pediatric patients treated with antidepressant drugs. Archives of general psychiatry. 2006; 63(3):332-339. [PubMed: 16520440]

9. Cipriani A, Zhou X, Del Giovane C, et al. Comparative efficacy and tolerability of antidepressants for major depressive disorder in children and adolescents: a network meta-analysis. Lancet. 2016 
10. Goldner EM, Jones W, Fang ML. Access to and waiting time for psychiatrist services in a Canadian urban area: a study in real time. Canadian journal of psychiatry Revue canadienne de psychiatrie. 2011; 56(8):474-480. [PubMed: 21878158]

11. Donaldson AE, Gordon MS, Melvin GA, Barton DA, Fitzgerald PB. Addressing the needs of adolescents with treatment resistant depressive disorders: a systematic review of rTMS. Brain Stimul. 2014; 7(1):7-12. [PubMed: 24527502]

12. Wall CA, Croarkin PE, Maroney-Smith MJ, et al. Magnetic Resonance Imaging-Guided, OpenLabel, High-Frequency Repetitive Transcranial Magnetic Stimulation for Adolescents with Major Depressive Disorder. J Child Adolesc Psychopharmacol. 2016; 26(7):582-589. [PubMed: 26849202]

13. McClintock SM, Reti IM, Carpenter LL, et al. Consensus Recommendations for the Clinical Application of Repetitive Transcranial Magnetic Stimulation (rTMS) in the Treatment of Depression. The Journal of clinical psychiatry. 2018; 79(1)

14. O'Reardon JP, Solvason HB, Janicak PG, et al. Efficacy and safety of transcranial magnetic stimulation in the acute treatment of major depression: a multisite randomized controlled trial. Biological psychiatry. 2007; 62(11):1208-1216. [PubMed: 17573044]

15. George MS, Lisanby SH, Avery D, et al. Daily left prefrontal transcranial magnetic stimulation therapy for major depressive disorder: a sham-controlled randomized trial. Archives of general psychiatry. 2010; 67(5):507-516. [PubMed: 20439832]

16. Croarkin PE, Rotenberg A. Pediatric Neuromodulation Comes of Age. J Child Adolesc Psychopharmacol. 2016; 26(7):578-581. [PubMed: 27604043]

17. Lisanby SH. Noninvasive Brain Stimulation for Depression - The Devil Is in the Dosing. The New England journal of medicine. 2017; 376(26):2593-2594. [PubMed: 28657865]

18. Grabb MC, Gobburu JVS. Challenges in developing drugs for pediatric CNS disorders: A focus on psychopharmacology. Progress in neurobiology. 2017; 152:38-57. [PubMed: 27216638]

19. Lefaucheur JP, Andre-Obadia N, Antal A, et al. Evidence-based guidelines on the therapeutic use of repetitive transcranial magnetic stimulation (rTMS). Clin Neurophysiol. 2014; 125(11):2150 2206. [PubMed: 25034472]

20. Brunoni AR, Chaimani A, Moffa AH, et al. Repetitive Transcranial Magnetic Stimulation for the Acute Treatment of Major Depressive Episodes: A Systematic Review With Network Metaanalysis. JAMA Psychiatry. 2017; 74(2):143-152. [PubMed: 28030740]

21. Li CT, Chen MH, Juan CH, et al. Efficacy of prefrontal theta-burst stimulation in refractory depression: a randomized sham-controlled study. Brain: a journal of neurology. 2014; 137(Pt 7): 2088-2098. [PubMed: 24817188]

22. Chung SW, Hoy KE, Fitzgerald PB. Theta-burst stimulation: a new form of TMS treatment for depression? Depress Anxiety. 2015; 32(3):182-192. [PubMed: 25450537]

23. Hunter AM, Cook IA, Greenwald SD, Tran ML, Miyamoto KN, Leuchter AF. The antidepressant treatment response index and treatment outcomes in a placebo-controlled trial of fluoxetine. Journal of clinical neurophysiology: official publication of the American Electroencephalographic Society. 2011; 28(5):478-482. [PubMed: 21946361]

24. Blumberger DM, Vila-Rodriguez F, Thorpe KE, et al. Effectiveness of theta burst versus highfrequency repetitive transcranial magnetic stimulation in patients with depression (THREE-D): a randomised non-inferiority trial. Lancet. 2018; 391(10131):1683-1692. [PubMed: 29726344]

25. Walter G, Tormos JM, Israel JA, Pascual-Leone A. Transcranial magnetic stimulation in young persons: a review of known cases. J Child Adolesc Psychopharmacol. 2001; 11(1):69-75. [PubMed: 11322748]

26. Loo C, McFarquhar T, Walter G. Transcranial magnetic stimulation in adolescent depression. Australasian psychiatry: bulletin of Royal Australian and New Zealand College of Psychiatrists. 2006; 14(1):81-85. [PubMed: 16630205]

27. Bloch Y, Grisaru N, Harel EV, et al. Repetitive transcranial magnetic stimulation in the treatment of depression in adolescents: an open-label study. The journal of ECT. 2008; 24(2):156-159. [PubMed: 18580562] 
28. Wall CA, Croarkin PE, Sim LA, et al. Adjunctive use of repetitive transcranial magnetic stimulation in depressed adolescents: a prospective, open pilot study. The Journal of clinical psychiatry. 2011; 72(9):1263-1269. [PubMed: 21951987]

29. Mayer G, Aviram S, Walter G, Levkovitz Y, Bloch Y. Long-term follow-up of adolescents with resistant depression treated with repetitive transcranial magnetic stimulation. The journal of ECT. 2012; 28(2):84-86. [PubMed: 22531199]

30. Le K, Liu L, Sun M, Hu L, Xiao N. Transcranial magnetic stimulation at 1 Hertz improves clinical symptoms in children with Tourette syndrome for at least 6 months. Journal of clinical neuroscience: official journal of the Neurosurgical Society of Australasia. 2013; 20(2):257-262. [PubMed: 23238046]

31. Yang XR, Kirton A, Wilkes TC, et al. Glutamate alterations associated with transcranial magnetic stimulation in youth depression: a case series. The journal of ECT. 2014; 30(3):242-247. [PubMed: 24820947]

32. Farzan F, Dhami P, Atluri S., et al. Effiacy and biological targets of theta burst stimulation in treatment of youth depression. 2nd Annual International Brain Stimulation Conference; 2017; Barcelona, Spain.

33. MacMaster FP, Croarkin PE, Wilkes TC. , et al. Biological Psychiatry. New York, NY: May 1, 2018 Open trial of repetitive transcranial magentic stimulation in youth with treatment-resistant major depression.

34. Walter G, Martin J, Kirkby K, Pridmore S. Transcranial magnetic stimulation: experience, knowledge and attitudes of recipients. The Australian and New Zealand journal of psychiatry. 2001; 35(1):58-61. [PubMed: 11270457]

35. Croarkin PE, Nakonezny PA, Wall CA, et al. Transcranial magnetic stimulation potentiates glutamatergic neurotransmission in depressed adolescents. Psychiatry Res. 2016; 247:25-33.

36. Krishnan C, Santos L, Peterson MD, Ehinger M. Safety of noninvasive brain stimulation in children and adolescents. Brain Stimul. 2015; 8(1):76-87. [PubMed: 25499471]

37. Davis NJ. Transcranial stimulation of the developing brain: a plea for extreme caution. Front Hum Neurosci. 2014; 8:600. [PubMed: 25140146]

38. Geddes L. Brain stimulation in children spurs hope - and concern. Nature. 2015; 525(7570):436437. [PubMed: 26399806]

39. Rossi S, Hallett M, Rossini PM, Pascual-Leone A. Safety, ethical considerations, and application guidelines for the use of transcranial magnetic stimulation in clinical practice and research. Clin Neurophysiol. 2009; 120(12):2008-2039. [PubMed: 19833552]

40. Perera T, George MS, Grammer G, Janicak PG, Pascual-Leone A, Wirecki TS. The Clinical TMS Society Consensus Review and Treatment Recommendations for TMS Therapy for Major Depressive Disorder. Brain Stimul. 2016; 9(3):336-346. [PubMed: 27090022]

41. McClintock SM, Reti IM, Carpenter LL, et al. Consensus Recommendations for the Clinical Application of Repetitive Transcranial Magnetic Stimulation (rTMS) in the Treatment of Depression. The Journal of clinical psychiatry. 2017

42. Wu CS, Liu HY, Tsai HJ, Liu SK. Seizure Risk Associated With Antidepressant Treatment Among Patients With Depressive Disorders: A Population-Based Case-Crossover Study. The Journal of clinical psychiatry. 2017; 78(9):e1226-e1232. [PubMed: 29068610]

43. Allen CH, Kluger BM, Buard I. Safety of Transcranial Magnetic Stimulation in Children: A Systematic Review of the Literature. Pediatric neurology. 2017; 68:3-17. [PubMed: 28216033]

44. Chiramberro M, Lindberg N, Isometsa E, Kahkonen S, Appelberg B. Repetitive transcranial magnetic stimulation induced seizures in an adolescent patient with major depression: a case report. Brain Stimul. 2013; 6(5):830-831.

45. Hu SH, Wang SS, Zhang MM, et al. Repetitive transcranial magnetic stimulation-induced seizure of a patient with adolescent-onset depression: a case report and literature review. The Journal of international medical research. 2011; 39(5):2039-2044. [PubMed: 22118010]

46. Cullen KR, Jasberg S, Nelson B, Klimes-Dougan B, Lim KO, Croarkin PE. Seizure Induced by Deep Transcranial Magnetic Stimulation in an Adolescent with Depression. J Child Adolesc Psychopharmacol. 2016; 26(7):637-641. [PubMed: 27447245] 
47. Therapy NA. [Accessed May 27, 2018, 2018] Safety and Effectiveness of NeuroStar® Transcranial Magnetic Stimulation (TMS) Therapy in Depressed Adolescents. 2015. https:// www.clinicaltrials.gov/ct2/show/NCT02586688

48. Dubin MJ, Mao X, Banerjee S, et al. Elevated prefrontal cortex GABA in patients with major depressive disorder after TMS treatment measured with proton magnetic resonance spectroscopy. Journal of psychiatry \& neuroscience: JPN. 2016; 41(3):E37-45. [PubMed: 26900793]

49. Croarkin PE. [Accessed May 27, 2018, 2018] Biomarkers in Repetitive Transcranial Magnetic Stimulation (rTMS) for Adolescent Depression. 2018. https://www.clinicaltrials.gov/ct2/show/ NCT03363919

50. Croarkin PE, Levinson AJ, Daskalakis ZJ. Evidence for GABAergic inhibitory deficits in major depressive disorder. Neurosci Biobehav Rev. 2011; 35(3):818-825. [PubMed: 20946914]

51. Sanacora G, Treccani G, Popoli M. Towards a glutamate hypothesis of depression: an emerging frontier of neuropsychopharmacology for mood disorders. Neuropharmacology. 2012; 62(1):6377. [PubMed: 21827775]

52. Vaiva G, Thomas P, Ducrocq F, et al. Low posttrauma GABA plasma levels as a predictive factor in the development of acute posttraumatic stress disorder. Biological psychiatry. 2004; 55(3):250254. [PubMed: 14744465]

53. Valle AC, Dionisio K, Pitskel NB, et al. Low and high frequency repetitive transcranial magnetic stimulation for the treatment of spasticity. Developmental medicine and child neurology. 2007; 49(7):534-538. [PubMed: 17593127]

54. Gabbay V, Mao X, Klein RG, et al. Anterior cingulate cortex gamma-aminobutyric acid in depressed adolescents: relationship to anhedonia. Archives of general psychiatry. 2012; 69(2):139149. [PubMed: 21969419]

55. Price JL, Drevets WC. Neurocircuitry of mood disorders. Neuropsychopharmacology. 2010; 35(1): 192-216. [PubMed: 19693001]

56. Croarkin PE, Nakonezny PA, Husain MM, et al. Evidence for increased glutamatergic cortical facilitation in children and adolescents with major depressive disorder. JAMA Psychiatry. 2013; 70(3):291-299. [PubMed: 23303429]

57. Gabbay V, Johnson AR, Alonso CM, Evans LK, Babb JS, Klein RG. Anhedonia, but not irritability, is associated with illness severity outcomes in adolescent major depression. J Child Adolesc Psychopharmacol. 2015; 25(3):194-200. [PubMed: 25802984]

58. Zhang W, Rosenkranz JA. Effects of Repeated Stress on Age-Dependent GABAergic Regulation of the Lateral Nucleus of the Amygdala. Neuropsychopharmacology. 2016; 41(9):2309-2323. [PubMed: 26924679]

59. Gilad GM, Gilad VH, Wyatt RJ, Tizabi Y. Region-selective stress-induced increase of glutamate uptake and release in rat forebrain. Brain research. 1990; 525(2):335-338. [PubMed: 1979236]

60. Lowy MT, Gault L, Yamamoto BK. Adrenalectomy attenuates stress-induced elevations in extracellular glutamate concentrations in the hippocampus. Journal of neurochemistry. 1993; 61(5):1957-1960. [PubMed: 7901339]

61. Fontella FU, Vendite DA, Tabajara AS, et al. Repeated restraint stress alters hippocampal glutamate uptake and release in the rat. Neurochemical research. 2004; 29(9):1703-1709. [PubMed: 15453265]

62. Ehrlich DE, Ryan SJ, Hazra R, Guo JD, Rainnie DG. Postnatal maturation of GABAergic transmission in the rat basolateral amygdala. Journal of neurophysiology. 2013; 110(4):926-941. [PubMed: 23719209]

63. Krnjevic K, Randic M, Straughan DW. Nature of a cortical inhibitory process. The Journal of physiology. 1966; 184(1):49-77. [PubMed: 4958616]

64. Krnjevic K, Randic M, Straughan DW. An inhibitory process in the cerebral cortex. The Journal of physiology. 1966; 184(1):16-48. [PubMed: 4958615]

65. Ziemann U. Pharmacology of TMS. Supplements to Clinical neurophysiology. 2003; 56:226-231. [PubMed: 14677399]

66. Ziemann U, Reis J, Schwenkreis P, et al. TMS and drugs revisited 2014. Clin Neurophysiol. 2015; 126(10):1847-1868. [PubMed: 25534482] 
67. Lewis CP, Nakonezny PA, Blacker CJ, et al. Cortical inhibitory markers of lifetime suicidal behavior in depressed adolescents. Neuropsychopharmacology. 2018

68. Lewis CP, Nakonezny PA, Ameis SH, et al. Cortical inhibitory and excitatory correlates of depression severity in children and adolescents. J Affect Disord. 2016; 190:566-575. [PubMed: 26580570]

69. Dyke K, Pepes SE, Chen C, et al. Comparing GABA-dependent physiological measures of inhibition with proton magnetic resonance spectroscopy measurement of GABA using ultra-highfield MRI. NeuroImage. 2017; 152:360-370. [PubMed: 28284797]

70. Lewis CP, Port JD, Frye MA, et al. An Exploratory Study of Spectroscopic Glutamatergic Correlates of Cortical Excitability in Depressed Adolescents. Frontiers in neural circuits. 2016; 10:98. [PubMed: 27965544]

71. Beam W, Borckardt JJ, Reeves ST, George MS. An efficient and accurate new method for locating the F3 position for prefrontal TMS applications. Brain Stimul. 2009; 2(1):50-54. [PubMed: 20539835]

72. Mir-Moghtadaei A, Caballero R, Fried P, et al. Concordance Between BeamF3 and MRIneuronavigated Target Sites for Repetitive Transcranial Magnetic Stimulation of the Left Dorsolateral Prefrontal Cortex. Brain Stimul. 2015; 8(5):965-973. [PubMed: 26115776]

73. Poznanski EO, Grossman JA, Buchsbaum Y, Banegas M, Freeman L, Gibbons R. Preliminary studies of the reliability and validity of the children's depression rating scale. Journal of the American Academy of Child Psychiatry. 1984; 23(2):191-197. [PubMed: 6715741] 


\section{KEY POINTS}

- $\quad$ Adolescent depression is a substantial global public health problem which contributes to academic failure, occupational impairment, deficits in social function, substance use disorders, teen pregnancy and completed suicide.

- Existing treatment approaches such as psychotherapy, pharmacotherapy, or combination treatment often have suboptimal results and uncertain safety profiles.

- Brain stimulation modalities such as transcranial magnetic stimulation have the potential for enduring, brain-based interventions for adolescents with depression.

- Existing work with transcranial magnetic stimulation in adolescents is nascent and larger, developmentally informed studies are needed.

- $\quad$ Treatment with transcranial magnetic stimulation may address imbalances in cortical GABAergic and glutamatergic neural circuitry. 


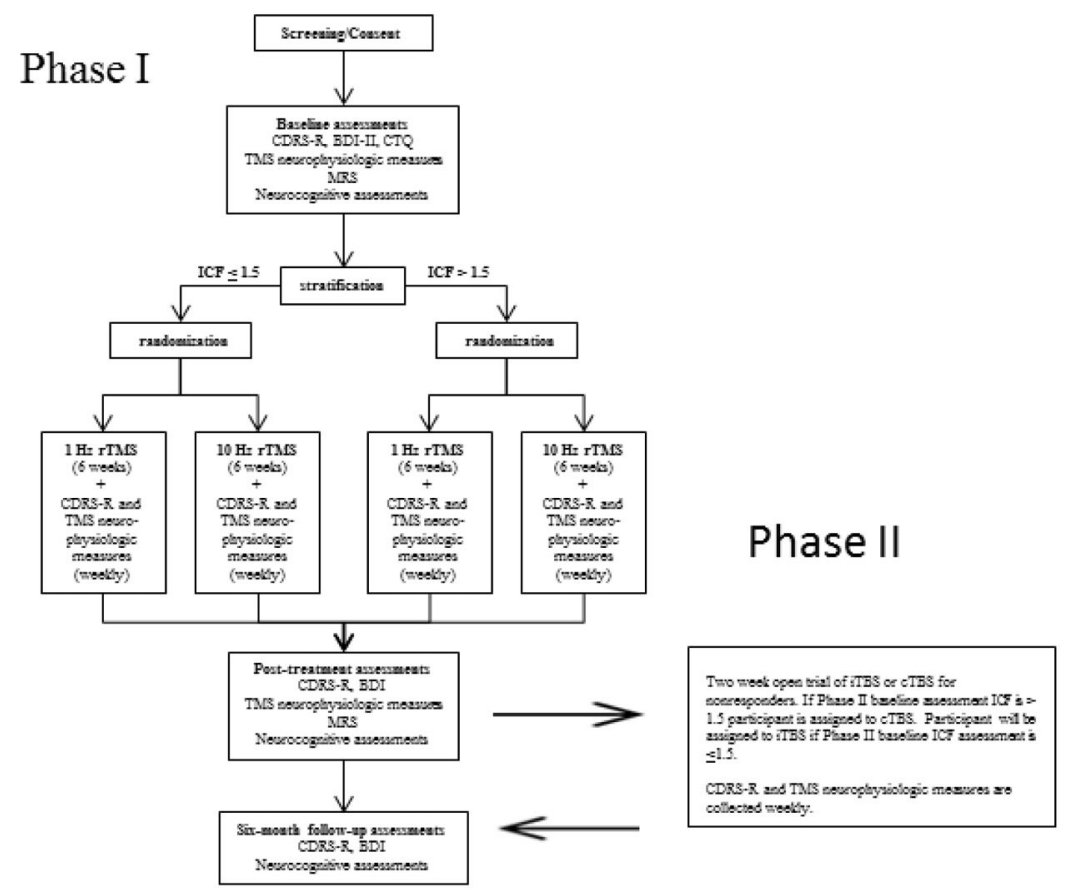

Figure 1.

Study schema 


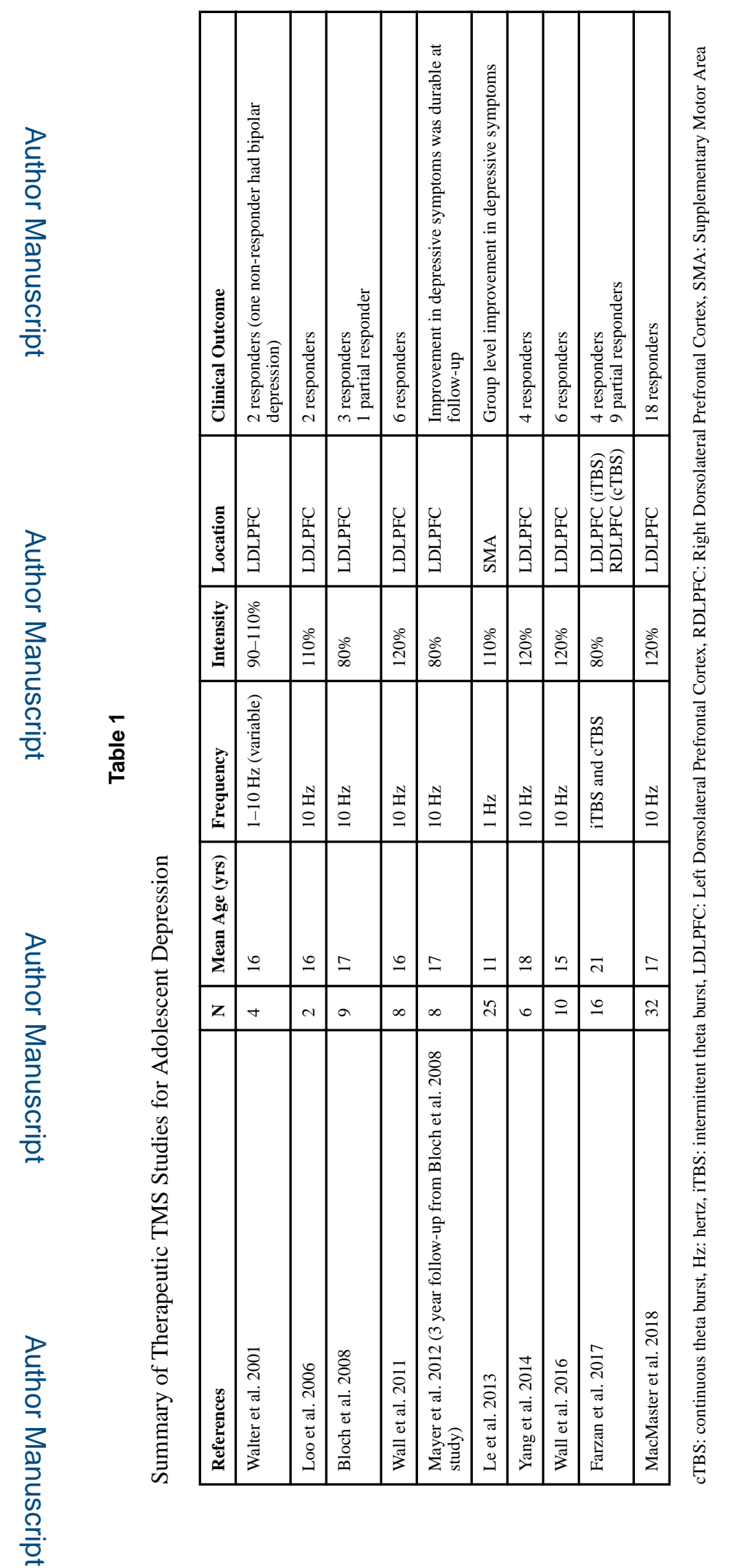

Child Adolesc Psychiatr Clin N Am. Author manuscript; available in PMC 2020 January 01. 